\title{
Stratéole/Vorcore-Long-duration, Superpressure Balloons to Study the Antarctic Lower Stratosphere during the 2005 Winter
}

\author{
Albert Hertzog,* Philippe Cocouerez, ${ }^{+}$Claude Basdevant, ${ }^{\#}$ Gillian Boccara, \\ Jérôme Bordereau, \# Bernard Brioit, ${ }^{\#}$ Alain Cardonne, ${ }^{@}$ René Guilbon, ${ }^{+}$Alain Ravissot, $@$ \\ Éric Schmitt, ${ }^{@}$ Jean-NoËl Valdivia, ${ }^{+}$Stéphanie Venel, ${ }^{+}$and François Vial ${ }^{\#}$ \\ * Laboratoire de Météorologie Dynamique, Université Pierre et Marie Curie, IPSL, CNRS, Palaiseau, France \\ + Centre National d'Études Spatiales, Toulouse, France \\ \# Laboratoire de Météorologie Dynamique, École Polytechnique, IPSL, CNRS, Palaiseau, France \\ @ Centre National d'Études Spatiales, Aire-sur-l'Adour, France
}

(Manuscript received 5 December 2006, in final form 4 April 2007)

\begin{abstract}
In September and October 2005, the Stratéole/Vorcore campaign flew 27 superpressure balloons from McMurdo, Antarctica, into the stratospheric polar vortex. Long-duration flights were successfully achieved, 16 of those flights lasting for more than 2 months. Most flights were terminated because they flew out of the authorized flight domain or because of energy shortage in the gondola. The atmospheric pressure (1-Pa precision) was measured every minute during the flights, whereas air temperature observations $(0.25-\mathrm{K}$ accuracy) and balloon positions (absolute GPS observations, 10-m accuracy) were obtained every 15 min. Fifteen-minute-averaged horizontal velocities of the wind were deduced from the successive balloon positions with a corresponding accuracy $\leqq 0.1 \mathrm{~m} \mathrm{~s}^{-1}$. The collected dataset (more than 150000 independent observations) provides a thorough high-resolution sampling of the polar lower stratosphere in the Southern Hemisphere from its wintertime state up to the establishment of the summer circulation in DecemberJanuary. Most of the balloons stayed inside the vortex until its final breakdown, although a few were ejected toward the midlatitudes in November during filamention events associated with an increase in planetary wave activity. The balloons behaved as quasi-Lagrangian tracers during the first part of the campaign (quiescent vortex) and after the vortex breakdown in early December. Large-amplitude mountain gravity waves were detected over the Antarctic Peninsula and caused one flight termination associated with the sudden burst in the balloon superpressure.
\end{abstract}

\section{Introduction}

Shortly after the discovery of the Antarctic ozone hole by Farman et al. (1985), the dynamical isolation of air parcels located well inside the stratospheric polarnight vortex was identified as a key factor in the processes that led to ozone depletion (McIntyre 1989). Since then, it has been acknowledged that besides chemistry, dynamics play a major role in determining the geographical structure, intensity, and variability of the ozone hole (e.g., WMO 2003, and references therein). In the late 1980s and beginning of the 1990s, however, the degree of isolation of air parcels located in

Corresponding author address: A. Hertzog, Laboratoire de Météorologie Dynamique, École Polytechnique, F-91128 Palaiseau CEDEX, France.

E-mail: hertzog@lmd.polytechnique.fr the inner vortex, as well as how they could eventually be transported to the midlatitude stratosphere and be irreversibly mixed there, was not fully understood. In this context, Vial et al. (1995) suggested that a set of quasi-Lagrangian, balloonborne observations could be very useful to document the dynamics and chemistry in the otherwise poorly observed Southern Hemisphere (SH) polar stratosphere in winter.

This project, named Stratéole, consisted in launching 200 superpressure balloons (SPBs) able to fly for a few months at about 50 and $70 \mathrm{hPa}$ in and around the polar vortex. Those balloons actually have the property to stay on constant-density (isopycnic) surfaces (e.g., Levanon et al. 1974), and thus to provide a good estimate of the motions of stratospheric air parcels that are isentropic to first order. In the 1960s-70s, a number of superpressure balloon flights already demonstrated the potential interest of those devices for scientific pur-

DOI: 10.1175/2007JTECHA948.1 
poses. For instance, the Global Horizontal Sounding Technique (GHOST; 1966-70), Éole (1971-72), and Global Atmospheric Research Programme (GARP)related Tropical Wind, Energy Conversion, and Reference Level Experiment (TWERLE; 1975) campaigns together flew hundreds of superpressure balloons in the SH upper troposphere (Lally et al. 1966; Morel and Bandeen 1973; TWERLE Team 1977). Flight durations longer than 3 months were frequently achieved. At the same time, about 50 stratospheric flights of superpressure balloons (float level between 50 and $18 \mathrm{hPa}$ ) were also performed (Angell 1972; Schumann 1980; Olivero et al. 1984). Though the dataset gathered during those early flights allowed the documenting of characteristics of the atmospheric dynamics that were unaccessible to the contemporary techniques, the lack of global communication and positioning systems significantly limited the observation frequency and accuracy. The situation changed in the 1980s, and Stratéole proposed to benefit from the assets of global-scale superpressure balloon flights and from modern, satelliteborne communication and positioning systems to provide an unprecedented in situ sampling of the polar stratosphere.

However, the project had to cope with a number of logistic and technological difficulties, and in the late 1990s it was decided to split Stratéole into two separate phases: the first one, called Vorcore, was devoted to the study of the vortex core and needed a few tens of balloons only. After several years of development and test campaigns, the Vorcore campaign finally took place in McMurdo, Antarctica, in August-October 2005. The original scientific objectives were refined in light of our current knowledge of the ozone hole, but the technological preparatory campaigns confirmed that the originality of the Vorcore observing system could provide new insights into stratospheric dynamics (Vial et al. 2001; Hertzog and Vial 2001; Hertzog et al. 2002b). More specifically, the Vorcore goals now include the study of gravity wave activity in the SH high latitudes, the characterization of the dispersion regime inside the vortex core, the quantification of ozone depletion rates in spring, and the assessment of the operational analysis accuracy.

This paper is aimed at presenting the observing system used during Vorcore, as well as the main features of the observation set that has been collected during the campaign. It is organized as follows. Section 2 describes the Vorcore balloons and briefly reviews their physics. The scientific and technological observations performed by the payload are also presented in this section. Section 3 is devoted to the campaign itself. It gives details on the flight configuration used during Vorcore, the set of flights performed during the campaign, and the various events that caused their terminations. Sec- tion 4 reviews more precisely the Vorcore dataset. In particular, the geographical distribution of the observations and the balloon behavior in the stratospheric flow are emphasized. The last section is devoted to the presentation of foreseen developments for long-duration superpressure balloon flights and to their possible implications for the study of the stratosphere.

\section{Observation technique}

\section{a. Superpressure balloons}

\section{1) BALLOON CHARACTERISTICS AND LAUNCHING} TECHNIQUE

The balloons used during Vorcore are helium filled and have a spherical-shaped, closed envelope. Although their design mainly relies on the main characteristics of the balloons used in the successful 1970s-80s experiments, a dedicated development program had nevertheless been necessary in order to extend the mission capability to winter polar flights and to improve the balloon reliability (Cocquerez et al. 2001). In particular, the envelopes were made of a trilaminate polyester/polyamide film especially designed for the experiment. This film provides high stiffness in flight conditions and is flexible enough at room temperature for manufacturing and packaging. The film stiffness was one of the key design requirements, since it ensures that the balloon volume will stay constant during the flight, even though the helium overpressure inside the balloon may vary (see, e.g., Fig. 1). The film also provides the needed levels of strength and gas tightness for achieving long-duration flights.

To sample two density levels in the stratosphere (roughly corresponding to the 50- and 70-hPa pressure surfaces), two balloon sizes were developed: 10- and 8.5-m diameter. This solution was preferred to using only 10-m balloons with some ballast in order to fly at a lower level, since this would have induced more stringent requirements on the gas tightness of the envelopes.

A new concept for suspending the payload to the envelope was developed. Whereas the Éole payloads were attached to the balloons with the help of a net, the major feature of this new system was to distribute the loads induced by the payload only at the bottom of the envelope.

Finally, the development program also focused on redefining the classical techniques for launching superpressure balloons. This last point was needed to successfully perform launches in the severe polar surface conditions in winter and early spring. In particular, a special effort was devoted to avoiding the need for large and costly dedicated ground infrastructures. 

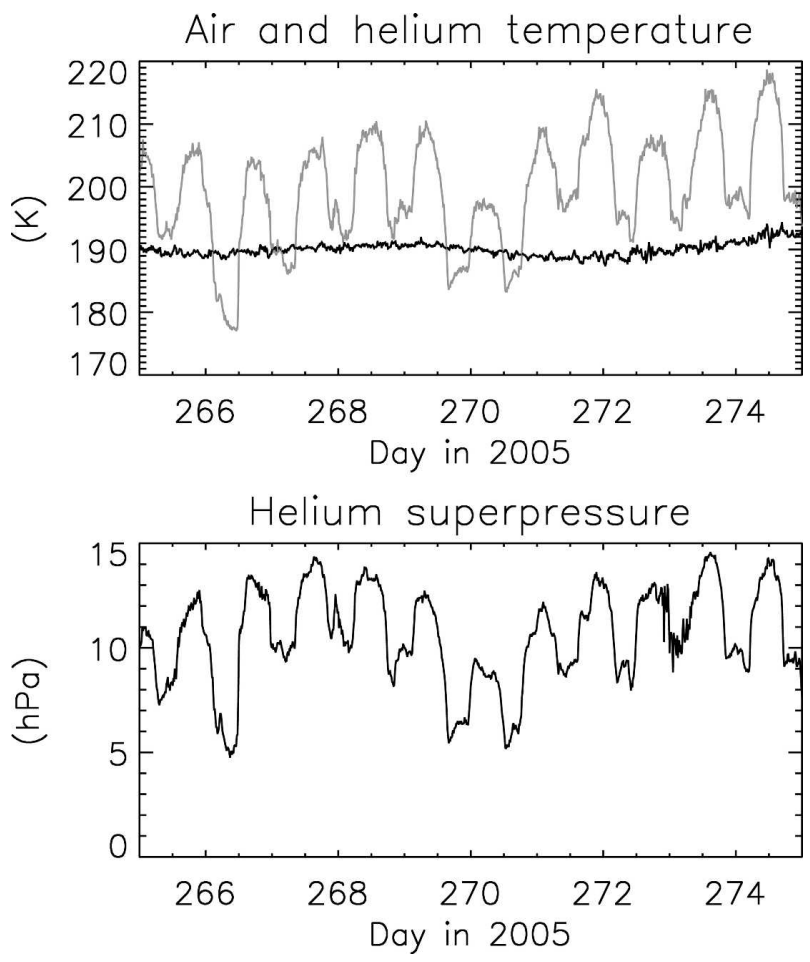

FIG. 1. (top) Air (black) and helium (gray) temperatures, and (bottom) helium superpressure observed during a section of Vorcore flight 4.

Namely, the balloons are now inflated while lying on an 18-m-long table that can easily be moved from the preparation building to the launchpad when the mean wind velocity is forecast to stay below $4 \mathrm{~m} \mathrm{~s}^{-1}$ for more than $30 \mathrm{~min}$. The inflation operations are performed outside, which allows the use of a small-height building (see Fig. 5). An inflation bench, based on a sonic-nozzle flowmeter, was also specially designed to work at very low temperatures.

\section{2) BALLOON PHYSICS}

One of the most critical parameters when flying superpressure balloons is to carefully determine the mass of lifting gas that fills the balloon. The gas amount actually controls the superpressure in the balloon, and one has to avoid both an excess of superpressure that would cause the balloon to burst and a vanishing superpressure that may destabilize the balloon. The maximum superpressure that the balloon can bear is theoretically computed from the mechanical properties of the envelope film and from a stress analysis. But the actual gas pressure during the flight (or, equivalently, the gas temperature once the balloon volume and gas amount are known) results from a complex radiativeconvective equilibrium between the gas, the balloon envelope, and the environment. This is exemplified in Fig. 1, which shows the helium and air temperatures as well as the superpressure during a 10-day period of one of the Vorcore flights. While the air temperature stays almost constant during that period, the helium temperature (and superpressure) exhibits large daily fluctuations caused by the variations in solar radiation. Furthermore, local drops in superpressure and gas temperature (like, e.g., around days 266 and 270) are caused by variations in the upward infrared radiation due to the presence of high-altitude clouds or to very cold surfaces (i.e., the Antarctic Plateau).

Therefore, before the campaign, a mission analysis based on climatological records of temperature, pressure, and radiative fluxes is performed so as to simulate the gas superpressure during the flights. The aim of this mission analysis is to determine the mass of helium that would statistically minimize the risk of balloon burst or depressurization. During Vorcore, this mass significantly varied from the early winter part of the flights to the end of the campaign in late spring, and a compromise between both flight conditions was adopted.

\section{3) BALloOn BEHAVIOR IN THE ATMOSPHERE}

The goal of the following paragraphs is not to give a complete review of the response of superpressure balloons to atmospheric motions, which has already been done theoretically and numerically elsewhere (e.g., Reynolds 1973; Massman 1978; Nastrom 1980; Hammam 1991). It is rather to recall a few characteristics of those balloon flights that may help to follow the discussions in the next sections. The behavior of superpressure balloons in the atmosphere can safely be decoupled into horizontal and vertical motions. On the horizontal, the most significant force is the aerodynamic drag that prevents the balloon from having a velocity much different than that of the wind. At first order, the equation of horizontal motions actually reads (Vial et al. 2001)

$$
\frac{d(\boldsymbol{v}-\mathbf{u})}{d t}=-L_{c}^{-1}|\boldsymbol{v}-\mathbf{u}|(\boldsymbol{v}-\mathbf{u}),
$$

where $\boldsymbol{v}-\mathbf{u}$ is the difference between the wind velocity and the balloon velocity, and $L_{c}$ is a characteristic length that scales as the balloon radius and equals $40 \mathrm{~m}$ for a 10-m-diameter balloon. Integrating Eq. (1) and averaging over a time period $T$ shows that the averaged-velocity difference cannot exceed $L_{c} / T$. During Vorcore, the balloon horizontal velocities are averaged over $15 \mathrm{~min}$, and the difference between the balloon and wind velocities over that time period is thus lower than $0.05 \mathrm{~m} \mathrm{~s}^{-1}$. Superpressure balloons can therefore 
be considered very accurate tracers of horizontal motions.

In contrast, superpressure balloons cannot follow the vertical motions of air parcels: while the balloons have a constant volume (and thus are constrained to stay on isopycnic surfaces), air parcels undergo adiabatic variations of their volume when moving upward or downward. The relative (vertical) displacement of isentropic and isopycnic surfaces therefore determines how Lagrangian superpressure balloons are. For instance, let us consider the response of both surfaces to a local increase in temperature $\delta T$. The associated increase in potential temperature reads

$$
\delta \theta=\frac{\partial \theta}{\partial T} \delta T
$$

and the corresponding vertical displacement of the isentrope is

$$
\delta \zeta_{\theta}=\frac{\delta \theta}{\frac{d \theta}{d z}}=\frac{\frac{\partial \theta}{d \theta}}{\frac{\partial \theta}{d z}} \delta T .
$$

Similarly, the vertical displacement of the isopycnic surface induced by this temperature increase reads

$$
\delta \zeta_{\rho}=\frac{\frac{\partial \rho}{\partial T}}{\frac{d \rho}{d z}} \delta T .
$$

Hence, the ratio of both displacements is obtained as

$$
r=\frac{\delta \zeta_{\theta}}{\delta \zeta_{\rho}}=\frac{\frac{\partial \theta}{\partial T} \frac{d \rho}{d \theta} \frac{d z}{d z}}{\frac{\partial \rho}{\partial T}} .
$$

Typical values relevant for the Vorcore campaign (i.e., pressure: $60 \mathrm{hPa}$, temperature: $200 \mathrm{~K}$, and BruntVäisälä frequency: $210^{-2} \mathrm{rad} \mathrm{s}^{-1}$ ) lead to $r \sim 4$, and air parcels typically undergo vertical displacements 4 times as large as those of the balloons.

Based on this simple calculus, Fig. 2 illustrates the motions of isentropic and isopycnic surfaces induced by a small-amplitude atmospheric wave. The disturbances in potential temperature observed by the balloons can be inferred from this figure: local coolings induce negative potential temperature disturbances in the balloon observations. The air parcels, on the other hand, evolve adiabatically and there are obviously no Lagrangian variations in potential temperature. The horizontal axis in Fig. 2 could also be the time in which case the figure

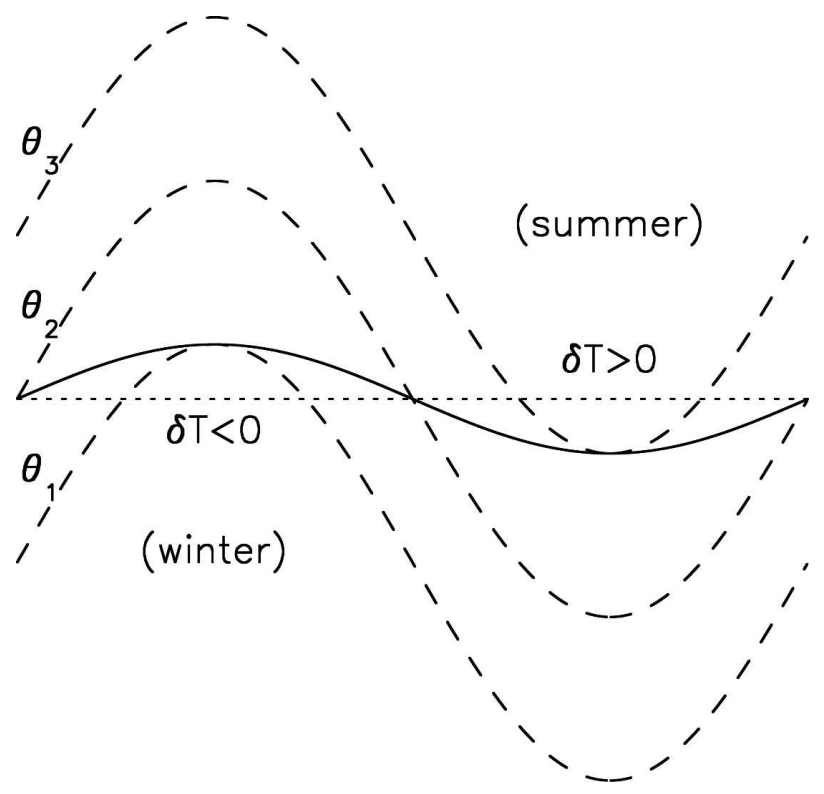

FIG. 2. Sketch of the relative displacement of isentropic surfaces (dashed) and isopycnic surfaces (solid) induced by an atmospheric wave (or by the annual cycle of stratospheric temperatures). Three isentropic surfaces (with $\theta_{1}<\theta_{2}<\theta_{3}$ ) are displayed. Superpressure balloons drift on isopycnic surfaces and "see" higher (lower) potential temperatures where the local Eulerian temperature disturbance is positive (negative). A horizontal dotted line is shown for reference.

grossly illustrates the annual cycle of stratospheric temperature at high latitudes. In winter, the temperatures are lower than the annual mean, and the transition from winter to summer is associated with an increase of potential temperatures in the balloon data.

A more complete calculation would, of course, consider the response of isopycnic and isentropic surfaces to pressure disturbances as well. On the one hand, atmospheric waves induce relative pressure disturbances that are one order of magnitude smaller than relative temperature disturbances. On the other hand, applying the same argument as above to a localized pressure disturbance shows that in that case both surfaces undergo almost similar displacements. The previous calculation and Fig. 2 therefore retain the essence of balloon motions with respect to those of air parcels.

\section{b. Payload}

The gondola (named Rumba) used during Vorcore performs the scientific and housekeeping observations, and checks that the balloon flight is safe. The gondola was designed to work in the very cold conditions met during Vorcore (air temperature lower than $-90^{\circ} \mathrm{C}$ ), so a 10-cm-thick polystyrene coating isolates the electronics from the environment. Furthermore, the electronics 
is heated during daytime through a resistor powered by solar generators. The electronics and sensors are powered by nonrechargeable batteries. The energy set was designed to allow 3-month flights, although the gondola consumption may slightly vary from one flight to the other. The gondola mass, including the coating, the batteries, the sensors devoted to atmospheric observations, as well as the straps for the mechanical connection with the flight train, is $\sim 10 \mathrm{~kg}$.

With the exception of atmospheric pressure, which is measured every minute, all the other observations are performed every $15 \mathrm{~min}$. The data are stored for $24 \mathrm{~h}$ in the gondola and sent to the ground via the satelliteborne Argos communication system. The communication bandwidth provided by Argos is very small (typically $30 \mathrm{~Kb} \mathrm{day}^{-1}$ ) and determines the sampling frequency. The various sensors used during Vorcore, as well as the processing performed to compensate for potential sensor biases, are now described.

\section{1) Pressure}

The barometer on board the Rumba gondola is a Paroscientific analog pressure transducer (model 216B) with an accuracy of $10 \mathrm{~Pa}$ and a precision of $1 \mathrm{~Pa}$. The barometer, which is located inside the gondola, is compensated in temperature down to $-54^{\circ} \mathrm{C}$. Temperatures inside the gondolas were very rarely colder than $-54^{\circ} \mathrm{C}$, and hence no further laboratory calibration of the barometers was needed.

The pressure is used to monitor the balloon vertical motions in the atmosphere. At the balloon flight level (i.e., atmospheric density $\sim 0.1 \mathrm{~kg} \mathrm{~m}^{-3}$ ), the $1-\mathrm{Pa}$ precision corresponds to a vertical position precision of $1 \mathrm{~m}$.

\section{2) Horizontal position AND wind velocities}

The balloon horizontal positions are obtained by absolute GPS measurements, with an accuracy of $15 \mathrm{~m}$. With the exception of a few hour-long flight segments at the beginning of the campaign where the temperatures inside the gondolas were very cold, GPS fixes were nominally obtained during the flights.

The horizontal wind velocities are deduced from the successive GPS positions by centered finite differences. Since positions are recorded every $15 \mathrm{~min}$, the accuracy on the 15-min-averaged balloon horizontal velocities is $0.02 \mathrm{~m} \mathrm{~s}^{-1}$. As shown previously, the difference between the balloon and wind horizontal velocities cannot be significantly larger than $0.05 \mathrm{~m} \mathrm{~s}^{-1}$ during that time interval. Estimates of the 15-min-averaged horizontal wind velocities are thus obtained with an accuracy better than $0.1 \mathrm{~m} \mathrm{~s}^{-1}$.

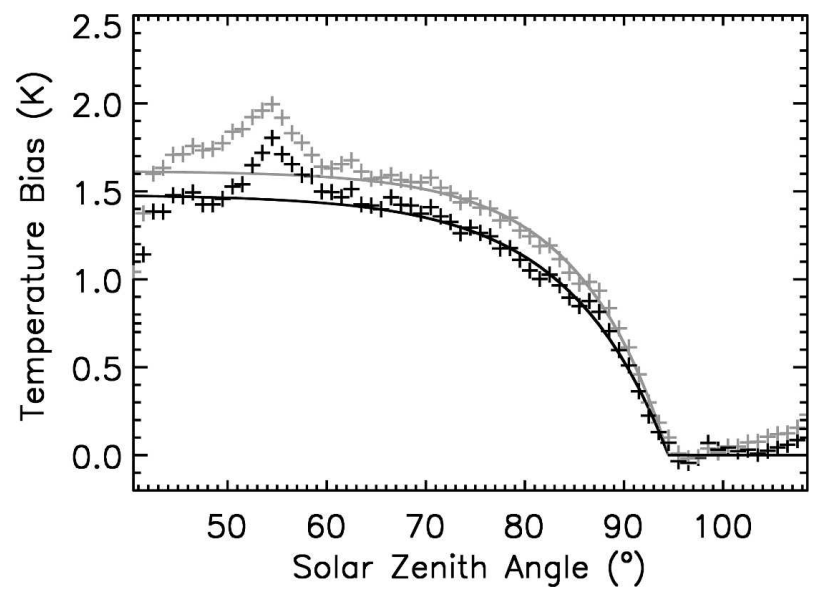

FIG. 3. Bias of temperature measurements (crosses) for one of the Vorcore flights (flight 13): first thermistor (black) and second thermistor (gray). Corrections subtracted from the raw temperature records (solid lines).

\section{3) Temperature}

The air temperature is measured by two independent, $120-\mu \mathrm{m}$-diameter microbead YSI thermistors that hang $5 \mathrm{~m}$ below the gondola. The thermistors are calibrated in the laboratory with respect to a standard Pt100 platine probe down to $-80^{\circ} \mathrm{C}$. To minimize the daytime radiative heating of the thermistor and its environment, thermistors are aluminized and mounted on small transparent glass plates (Fourrier et al. 1970).

The accurate measure of temperature on superpressure balloon flights is, however, particularly difficult: in contrast to classical radiosoundings that continuously ascend in the atmosphere, superpressure balloons move with the wind, and the temperature sensors are poorly ventilated. It has thus been observed that Rumba temperature measurements are warm biased during the day. This is exemplified in Fig. 3, which shows the temperature measurement bias versus solar zenith angles (SZAs) for one of the Vorcore flights. As detailed in Hertzog et al. (2004), that curve is obtained empirically by first computing the derivative of the temperature bias with respect to SZA and then integrating it. In the case of the Vorcore campaign, most of the observations were made at high SZAs, and the temperature bias estimates are therefore more accurate at those solar angles.

Figure 3 clearly shows that temperature observations are warmer during day than during night since no diurnal cycle with such an amplitude is expected in the lower stratosphere. The daytime bias may slightly vary from one thermistor to the other due to the proximity of the microbead with the glass plate or to the amount of metal deposited on the plate for the electrical con- 
nection between the thermistor and the gondola. However, most of the thermistors exhibit a $\sim 1.5-\mathrm{K}$ bias at low SZAs. An exponential is used to fit the daytime bias of each thermistor and then substracted from the raw temperature measurements.

After correction, the accuracy of temperature measurements, which can be estimated with the two independent temperature measurements performed on each flight, is found to be $0.25 \mathrm{~K}$ during the night and $0.3 \mathrm{~K}$ during the day. The temperature accuracy can be further improved by a factor $\sqrt{2}$ by taking the mean of both thermistor measurements.

\section{4) HouseKeEPing PARAMETERS}

Apart from the previous measurements that are intended for scientific use, Rumba also performs housekeeping observations. In brief, the balloon superpressure and gas temperature are measured by a Honeywell pressure tranducer and a 240- $\mu \mathrm{m}$-diameter YSI thermistor, with respective accuracies of $10 \mathrm{~Pa}$ and $0.5 \mathrm{~K}$. The gondola internal temperature, as well as the battery voltage, is also monitored during the flight.

\section{Vorcore experiment}

\section{a. Flight safety and configuration}

The flight configuration used during Vorcore is shown in Fig. 4. The thermistors are at the bottom of the flight train to avoid the balloon wake. A radar reflector and a flash lamp are used to signalize the flight train when it crosses the airplane space during the ascent and final descent. The end of the flight can be automatically commanded by the gondola as soon as one of the following safety criteria is no longer met:

- flight domain: the balloon must stay south of $40^{\circ} \mathrm{S}$,

- altitude: the balloon has to fly above $100 \mathrm{hPa}$,

- energy: the battery voltage has to be higher than a predetermined threshold,

- flight length: a maximum lifetime of 110 days is allowed.

In that case, the flight train is separated from the balloon and descends with a parachute. The unballasted balloon rapidly ascends and explodes because of the superpressure increase. The flight domain and altitude limitations have been imposed to minimize the probability of flying above populated areas or through airplane corridors. Finally, a secondary redundant device located just above the parachute is also able to

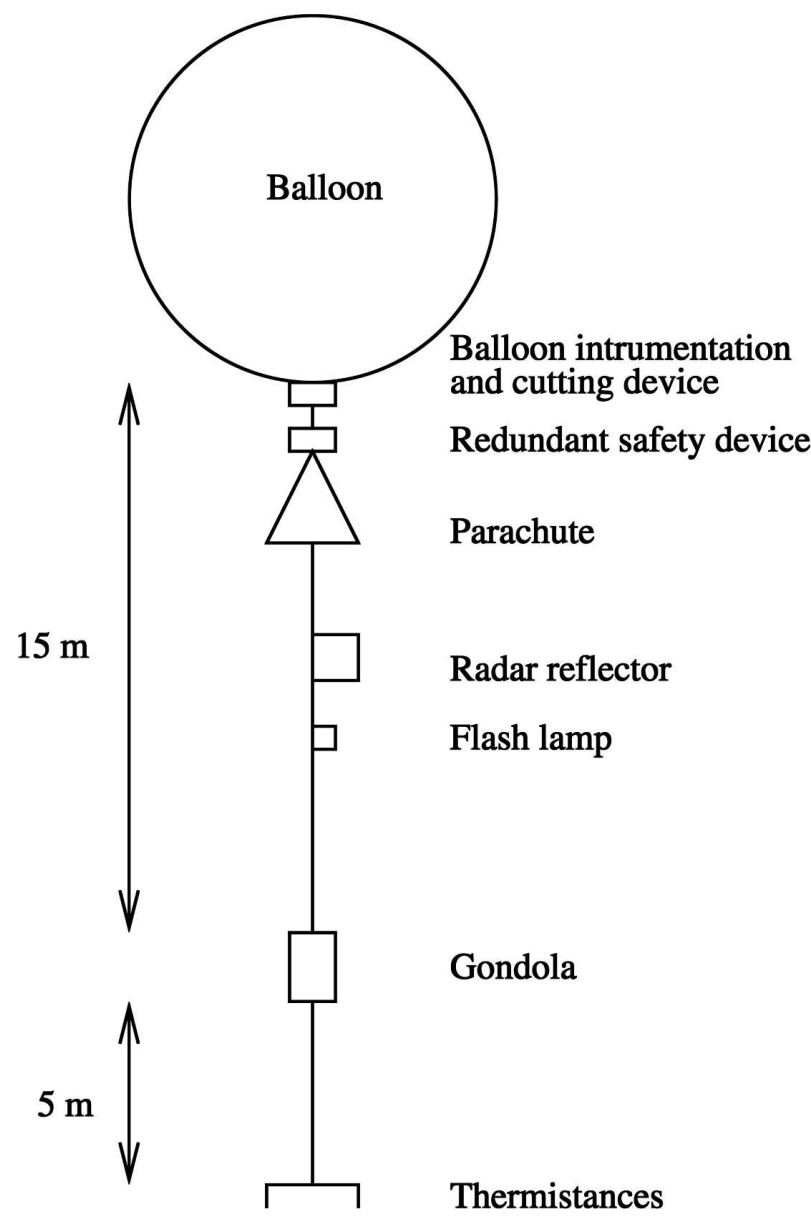

FIG. 4. The flight train configuration during Vorcore.

check the altitude and flight length criteria and to command the flight termination.

\section{b. Balloon campaign}

The Vorcore campaign took place in McMurdo, Antarctica. McMurdo is actually the only Antarctic basis south of $60^{\circ} \mathrm{S}$ that can be reached during winter and that provides sufficient logistic capabilities to support such a large balloon campaign. The launching site $\left(77.85^{\circ} \mathrm{S}, 166.65^{\circ} \mathrm{E}\right)$ was located on the sea ice, $300 \mathrm{~m}$ in front of the main station, in order to be as much protected as possible from the prevailing southeasterly surface winds that descend from the Antarctic Plateau and circulate around Ross Island. Figure 5 shows the launchpad that consisted of two 25-m-long, semicylindrical "Jamesways" in which the balloons were prepared before launch, two power generators, a few containers, and a regularly desnowed, $100 \mathrm{~m} \times 100 \mathrm{~m}$ squared area for the launches.

The first launch took place on 5 September and the last one on 28 October. A total of 27 launches were 


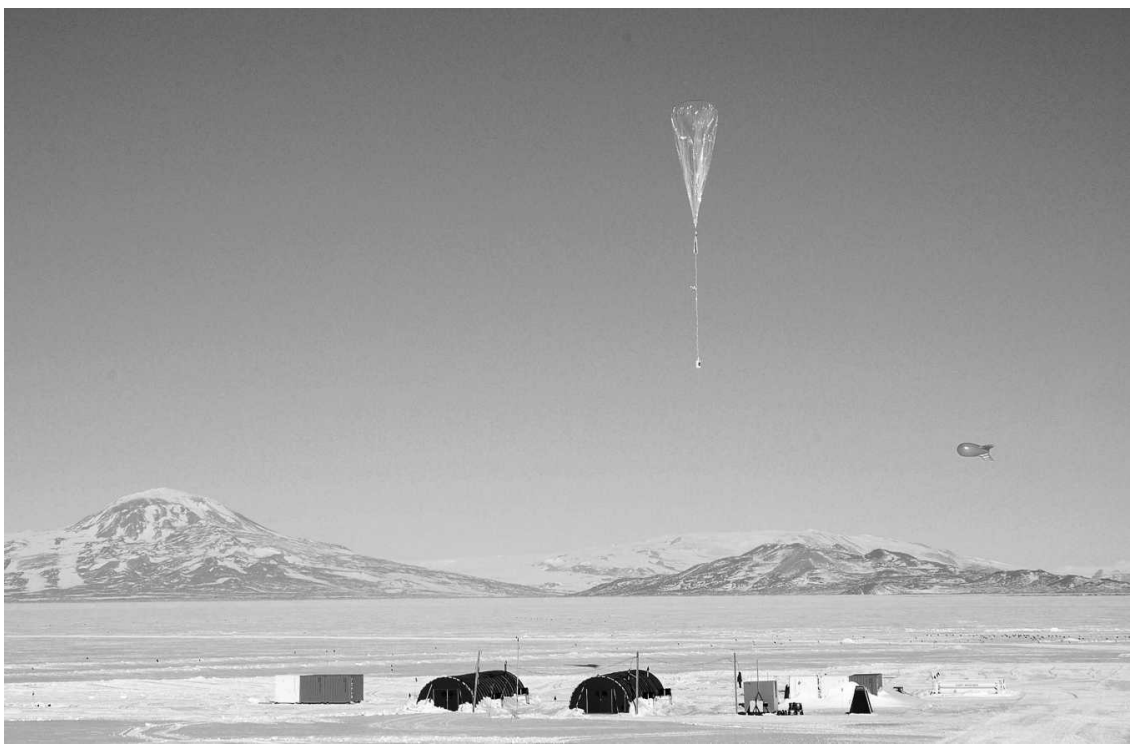

FIG. 5. A 10-m superpressure balloon just after launch. The Vorcore launchpad, with the two dark Jamesways used as preparation buildings, was located $300 \mathrm{~m}$ in front of McMurdo on the sea ice.

performed during this period. The weather limitations, imposed so as to minimize the risk of damaging the balloon during the final operational phases (i.e., mean surface-wind velocity smaller than $4 \mathrm{~m} \mathrm{~s}^{-1}$ and gusts smaller than $6 \mathrm{~m} \mathrm{~s}^{-1}$ ), somewhat limited the number of launch opportunities. However, windows long enough to launch the balloons were found on average $66 \%$ of the days in September and October. Two consecutive launches were even performed on seven occasions when calm surface conditions lasted for more than $3 \mathrm{~h}$.

Details on the 27 flights are given in Table 1. The mean flight duration achieved during the campaign is about 2 months (58.5 days), while the longest flight (flight 8) lasted 109 days. Gondolas running out of energy or balloons crossing the flight-domain boundary were the two main causes of flight terminations. Premature ends of the flights were otherwise principally caused by leaking balloon envelops (3 flights), balloon bursts due to a weakness of the envelops ( 2 flights), and balloon bursts due to atmospheric gravity waves with dramatic amplitudes (2 flights), as shown below.

Figure 6 shows the number of balloons in flight versus time. The launching period extended from the beginning of September to the end of October 2005, at which point a maximum of 21 balloons in flight was reached. In December 2005, this number rapidly fell due to the progressive breakdown of the stratospheric vortex that pushed the balloons toward the limits of the flight domain. Each balloon/gondola typically performs
100 observations per day, so that a total of 150743 observations were finally gathered during the campaign.

\section{c. Complementary observations}

Complementary observations associated with the Vorcore campaign have been performed in several Antarctic stations to complement the balloon observations. In particular, an emphasis has been put on ozone observations since no such observations are performed by the Vorcore gondola. To this end, ozone sondes were launched on alert in Davis $\left(68.58^{\circ} \mathrm{S}, 77.97^{\circ} \mathrm{E}\right)$, Marambio $\left(64.24^{\circ} \mathrm{S}, 56.66^{\circ} \mathrm{W}\right)$, McMurdo, and Neumayer $\left(70.65^{\circ} \mathrm{S}, 8.25^{\circ} \mathrm{W}\right)$ when superpressure balloons were flying close by. The balloon trajectories in conjunction with the ozone profiles will help to study the ozone depleting rates in the vortex core.

Rayleigh lidar observations to document the presence of polar stratospheric clouds were also performed in Davis, Dumont D'Urville $\left(66.67^{\circ} \mathrm{S}, 140.01^{\circ} \mathrm{E}\right)$, and McMurdo. Finally, on several occasions when Vorcore balloons were launched from or were flying above McMurdo, a high-resolution vertical profile of aerosol was obtained by the University of Wyoming aerosol counter (Deshler et al. 2003). These aerosol data will be used with the parcel-based history of air temperature provided by superpressure balloons to assess our understanding of microphysical processes in the polar stratosphere. 
TABLE 1. Summary of Vorcore flights. Flights associated with the same Greek letter were launched consecutively.

\begin{tabular}{|c|c|c|c|c|c|}
\hline Flight No. & Launch & End & Duration (day) & Balloon diameter (m) & Reason for flight termination \\
\hline 1 & 5 Sep 2005 & 11 Sep 2005 & 6 & 10 & Leaking balloon \\
\hline 2 & 6 Sep 2005 & 6 Dec 2005 & 90 & 10 & Energy \\
\hline $3^{\alpha}$ & 9 Sep 2005 & 5 Dec 2005 & 86 & 10 & Energy \\
\hline $4^{\alpha}$ & 9 Sep 2005 & 6 Nov 2005 & 57 & 8.5 & Flight domain \\
\hline 5 & 12 Sep 2005 & 27 Sep 2005 & 15 & 8.5 & Leaking balloon \\
\hline $6^{\beta}$ & 17 Sep 2005 & 17 Sep 2005 & 0 & 10 & Incident on flight train \\
\hline $7^{\beta}$ & 17 Sep 2005 & 18 Dec 2005 & 91 & 10 & Flight domain \\
\hline 8 & 20 Sep 2005 & 7 Jan 2006 & 109 & 8.5 & Flight domain \\
\hline $9^{\gamma}$ & 22 Sep 2005 & 24 Sep 2005 & 2 & 8.5 & Balloon burst \\
\hline $10^{\gamma}$ & 22 Sep 2005 & 17 Dec 2005 & 87 & 10 & Flight domain \\
\hline 11 & 23 Sep 2005 & 26 Dec 2005 & 95 & 8.5 & Energy \\
\hline 12 & 23 Sep 2005 & 8 Oct 2005 & 13 & 10 & Mountain wave \\
\hline 13 & 26 Sep 2005 & 13 Dec 2005 & 79 & 8.5 & Energy \\
\hline 14 & 28 Sep 2005 & 26 Dec 2005 & 89 & 10 & Energy \\
\hline 15 & 4 Oct 2005 & 7 Dec 2005 & 64 & 10 & Communication lost \\
\hline 16 & 5 Oct 2005 & 5 Jan 2006 & 93 & 8.5 & Energy \\
\hline $17^{\delta}$ & 5 Oct 2005 & 2 Nov 2005 & 28 & 10 & Leaking balloon \\
\hline $18^{\delta}$ & 6 Oct 2005 & 20 Dec 2005 & 75 & 10 & Flight domain \\
\hline $19^{\varepsilon}$ & 14 Oct 2005 & 14 Jan 2006 & 93 & 10 & Energy \\
\hline $20^{\varepsilon}$ & 14 Oct 2005 & 30 Oct 2005 & 16 & 10 & Mountain wave \\
\hline 21 & 14 Oct 2005 & 14 Oct 2005 & 0 & 8.5 & Balloon burst \\
\hline 22 & 16 Oct 2005 & 23 Dec 205 & 68 & 10 & Flight domain \\
\hline 23 & 18 Oct 2005 & 24 Dec 2005 & 66 & 10 & Flight domain \\
\hline $24^{\zeta}$ & 25 Oct 2005 & 27 Jan 2006 & 94 & 10 & Energy \\
\hline $25^{\zeta}$ & 25 Oct 2005 & 15 Dec 2005 & 51 & 10 & Flight domain \\
\hline $26^{\eta}$ & 28 Oct 2005 & 15 Nov 2005 & 17 & 10 & Flight domain \\
\hline $27^{\eta}$ & 28 Oct 2005 & 1 Feb 2006 & 96 & 10 & Energy \\
\hline
\end{tabular}

\section{Some characteristics of the Vorcore dataset}

\section{a. Geographical sampling}

Figure 7 shows the geographical distribution of the Vorcore observations. Because of the nonstationarity of the stratospheric flow and the long duration of the flights, the balloon flotilla thoroughly sampled the whole Antarctic continent and the ocean up to $55^{\circ} \mathrm{S}$.

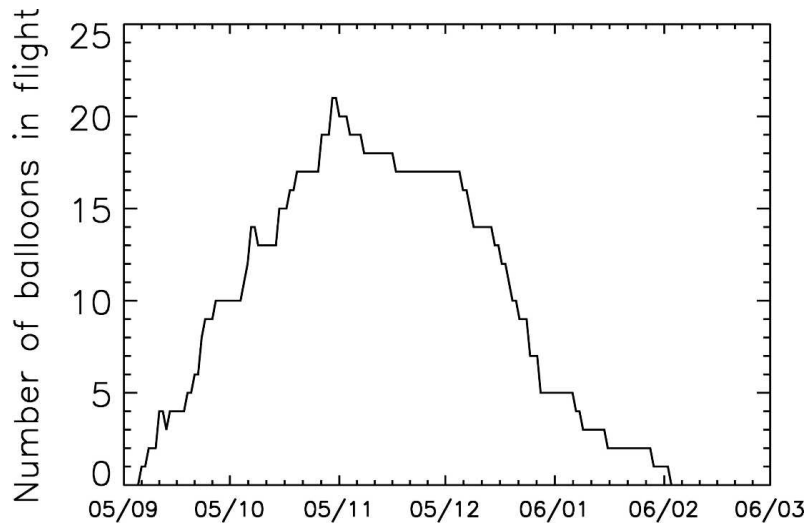

FIG. 6. Number of balloons in flight during the Vorcore campaign (September 2005-February 2006).
Note also that the cloud of observations is slightly displaced toward the Atlantic Ocean due to the preferred position of the 2005 vortex.

This apparently almost homogeneous sampling nevertheless hides varying geographical features of the observation set associated with the evolution of the stratospheric vortex in the 2005 winter and spring. Those features can be roughly divided into three periods, as shown in Fig. 8. At the beginning of the campaign (September-October), the stratospheric vortex was very stable and the flow was almost symmetric about the Pole. The observations performed during this period are associated with almost circular trajectories around the vortex center. In November, the vortex began to be stretched and displaced off the Pole by planetary waves with increasing amplitudes, and the sampling of the stratosphere slightly extends to the midlatitudes. During this month, the vortex center was most of the time located either in the Atlantic or in the western Indian sector of Antarctica, so that the balloons only occasionally flew above the Antarctica Pacific sector. Finally, in December and at the beginning of 2006, the stratospheric vortex broke into several pieces and most of the remaining balloons were ejected toward midlatitudes where some eventually crossed the $40^{\circ} \mathrm{S}$ parallel. 


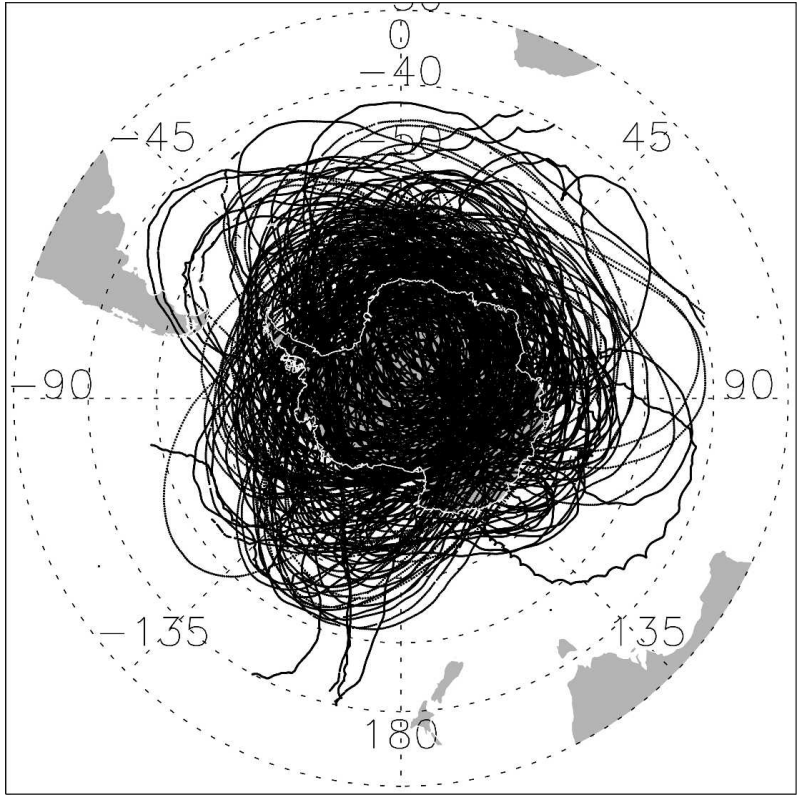

FIG. 7. Geographical distribution of Vorcore balloon observations. The flight domain was limited to latitudes southward of $40^{\circ} \mathrm{S}$

Thanks to the long flight duration achieved during the campaign, the Vorcore balloon flotilla therefore documented the $2005 \mathrm{SH}$ stratospheric vortex from its very stable winter state up to its total breakdown in early December.

\section{b. Observations with respect to the stratospheric vortex}

To monitor the positions of the balloons with respect to the vortex, the equivalent latitude of each observation has been computed. The equivalent latitude is the latitude of the parallel that encircles the same polar-cap area as the potential vorticity (PV) contour that passes by the balloon position. The $90^{\circ}$ equivalent latitude thus corresponds to the point with the lowest PV in the Southern Hemisphere vortex (the vortex center), while lower equivalent latitudes correspond to midlatitude air. The vortex edge is associated with a strong horizontal PV gradient on an isentropic surface (McIntyre and Palmer 1984), and is therefore computed as the extremum of the first derivative of PV with respect to equivalent latitude (Nash et al. 1996). Here, we used the PV fields provided by the European Centre for Medium-Range Weather Forecasts (ECMWF) operational analyses on 60 vertical levels at $0.5^{\circ} \times 0.5^{\circ}$ horizontal resolution.

The differences between the balloon equivalent latitudes and the vortex-edge equivalent latitude are shown in Fig. 9. As mentioned in the introduction, the aim of the Vorcore campaign was primarily to document the small to mesoscale dynamics in the core of the polar stratospheric vortex. Launches were consequently restricted to time periods when the vortex was located above the McMurdo station, and the balloon initial positions are located inside the vortex. As good tracers of air parcel motions, the superpressure balloons did not cross the dynamical barrier associated with the vortex edge during the first two months of the campaign. From October on, nevertheless, the progressive shift of the Vorcore observations toward the vortex edge reflects the regular shrinking of the vortex area that usually occurs in spring. In December, as shown in Fig. 8, the vortex splits in several pieces and finally disappears. At that time, a number of balloons are still located inside the remaining low-PV regions, and the Vorcore observations sample both sides of the vanishing vortices. However, some of the balloons escaped the vortex as soon as mid-November (i.e., much before the vortex breakdown). This was the case of balloon 27, whose positions between 20 and 27 November are reported in Fig. 10. This balloon, which was located close to the vortex edge at the beginning of the period, was embedded in a PV filament that was ultimately ejected from the main vortex. Those kinds of vortex erosion events are responsible for most of the exchange of air masses between the stratospheric vortex and the "surf zone" and are caused by breaking planetary-scale Rossby waves (McIntyre and Palmer 1983; Waugh et al. 1994; Koh and Plumb 2000; Moustaoui et al. 2003). After 23 November, balloon 27, though outside of the vortex, was still drifting in an air mass with a PV value lower than its surrounding, midlatitude air, and which is furthermore connected by a thin filament to the main vortex. During this period therefore, the balloon stayed in the air mass ejected from the vortex and thus achieved a Lagrangian sampling of the atmosphere. This quasi-Lagrangian aspect of superpressure balloon flights is further discussed in the next section.

\section{c. Quasi-Lagrangian observations}

Superpressure balloons do not drift on isentropic surfaces and as such are not perfect tracers of air masses in the stratosphere. Note, however, that although diabatic heating rates are quite low in the stratosphere (typically on the order of $1 \mathrm{~K}$ day $^{-1}$ ), they cannot be neglected on time scales corresponding to long-duration flights, so that even a pure isentropic balloon would not be a perfect tracer of air motion. The above example of balloon 27 nevertheless shows that at 


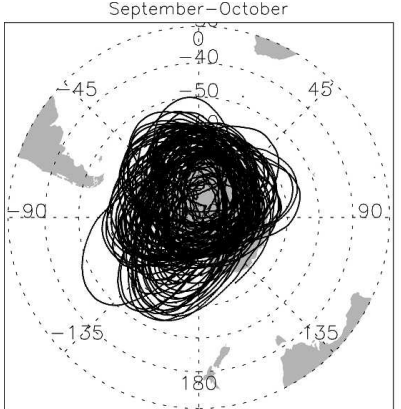

Oct 3, 2005

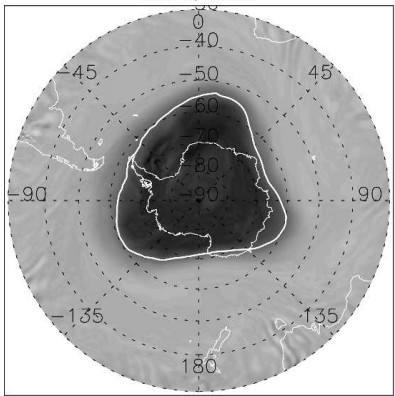

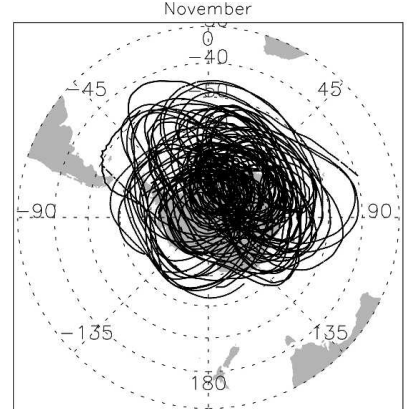

Nov 18,2005

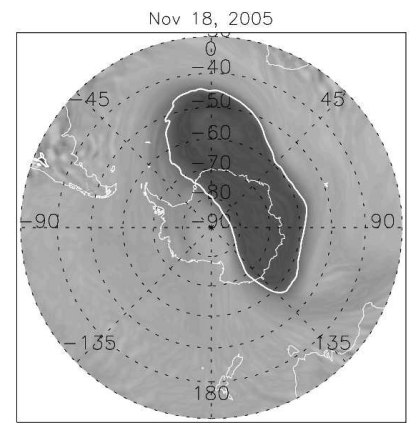

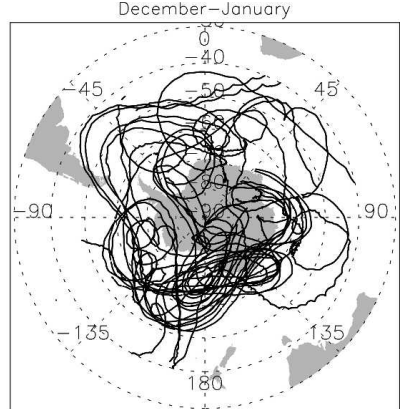

Dec 10, 2005

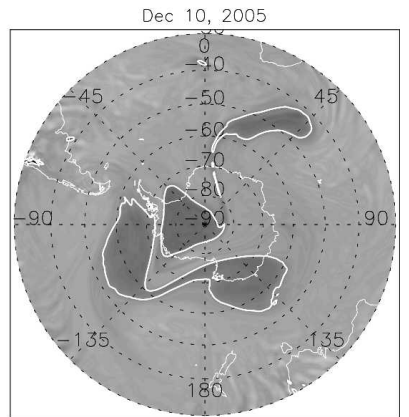

FIG. 8. (top) Geographical distribution of Vorcore balloon observations in (left) September-October, (middle) November, and (right) December-January. (bottom) Potential vorticity analyzed by ECMWF on the $475-\mathrm{K}$ isentrope on days representative of the three phases of the stratospheric flow during Vorcore: (left) centered vortex, (middle) vortex displaced off the South Pole but still well defined, and (right) vortex broken up in several pieces. The thick white line represents the vortex edge as defined by the Nash et al. (1996) algorithm. The PV color code is the same on the three panels.

least on short time scales, superpressure balloon trajectories mimic air-parcel trajectories to a good approximation. To further investigate the quasi-Lagrangian aspect of the Vorcore dataset, the air potential temperature measured along two balloon trajectories that are representative of the whole Vorcore flotilla is shown in Fig. 11.

In September 2005, the potential temperature of balloon 3 only slightly varies, so that this balloon almost

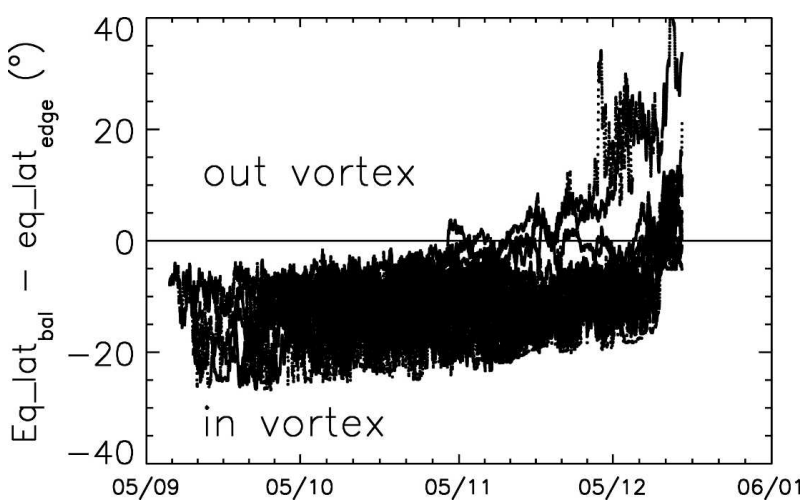

FIG. 9. Difference between the balloon equivalent latitudes and the vortex-edge equivalent latitude. permanently flies on the same isentropic surface. In fact, at that time, the undisturbed state of the vortex causes isentropic and isopycnic surfaces to be roughly parallel to each other in the lower stratosphere. The balloons can therefore be considered Lagrangian tracers to a good approximation at the beginning of the experiment. In October 2005, Fig. 11 shows that balloon 3 potential temperature increases from $\sim 450$ up to $480 \mathrm{~K}$. This increase results from the progressive warming of the vortex core with the return of the sun and the associated differential displacements of isentropic and isopycnic surfaces. In November, planetary-scale Rossby waves induce large variations of the balloon potential temperatures (up to $30-\mathrm{K}$ amplitude), so that the quasi-Lagrangian property of the balloon flotilla is more arguable from mid-October to the beginning of December. Finally, once the balloons escape from the main vortex (as for balloon 27) or after the vortex final breakdown, the potential temperature along the balloon trajectories stays almost constant again. In that last case, the balloons flew in the vicinity of the zero-wind altitude where Rossby waves become evanescent. Once again, the balloons can thus be considered good Lagrangian tracers, which is, for instance, exemplified by balloon 27 (cf. Fig. 10). 


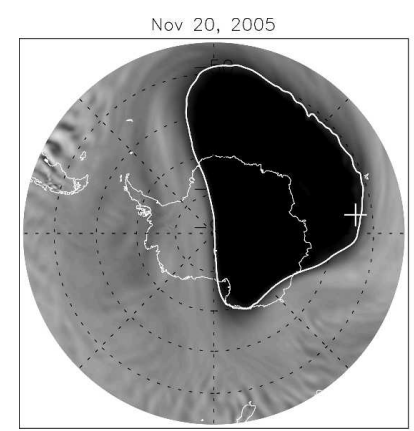

Nov 23, 2005
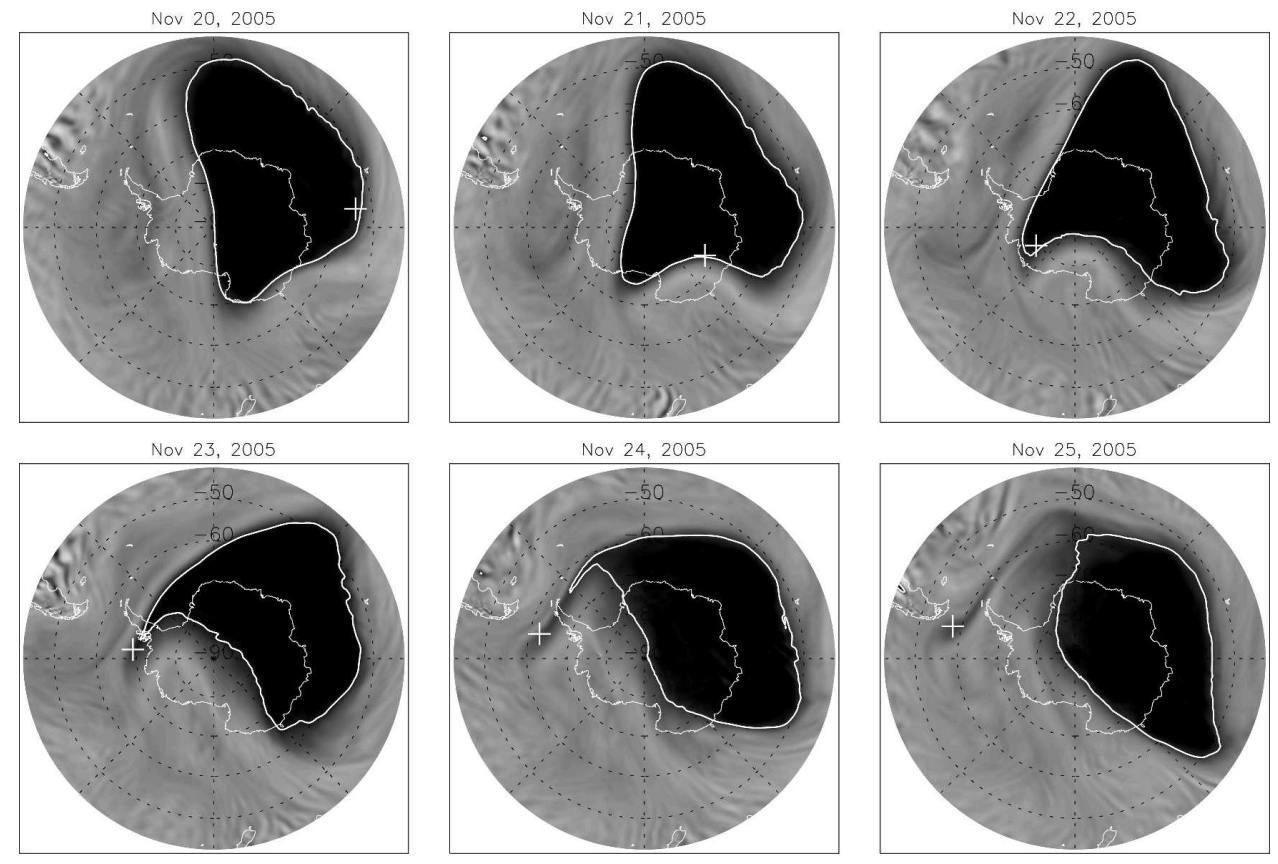

Nov 25, 2005

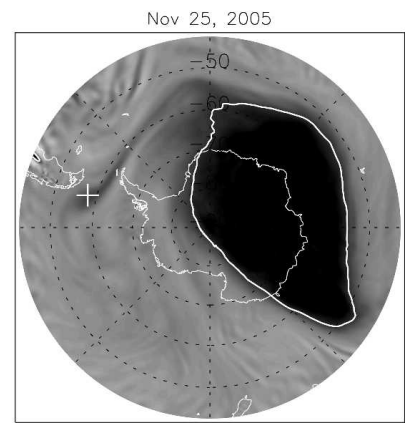

FIG. 10. Potential vorticity on the 475-K isentrope analyzed by ECMWF at 0000 UTC from 20 to 25 Nov 2005. Dark (light) colors are associated with lower (higher) PV values. The thick white line represents the vortex edge. The white cross shows balloon 27's position at 0000 UTC on the corresponding day.

\section{d. An example out of the dataset: The end of flight 12}

In this section, the observations performed by flight 11 on 7 October 2005 are analyzed to (i) present the kind of motions that can be studied with the dataset collected during Vorcore and (ii) show how strong atmospheric waves can induce dramatic damages to superpressure balloons. Flights 11 and 12 were launched $20 \mathrm{~h}$ apart on 22 September 2005, but were still flying close to each other 12 days after the launch, flight 11 leading flight 12 by about $4 \mathrm{~h}$. The trajectories of both balloons on 6 and 7 October 2005, while the balloons were flying above the northern tip of the Antarctic Peninsula, are shown in Fig. 12. Until 1000 UTC on 7 October, the gondola on board flight 12 was in the nominal flight mode, reporting data every $15 \mathrm{~min}$, and all the balloon and payload housekeeping parameters were nominal. At that time, the balloon was very close to the western coast of the Antarctic Peninsula (see Fig. 12). The next point received from this balloon indicates that the gondola had crossed the 100-hPa flight-domain limit. This point is located on the eastern side of the Antarctic Peninsula, and the altitude reported by the GPS was $3000 \mathrm{~m}$. The vertical velocity of the payload was negative and consistent with a descent under the parachute. The last point received from flight 12 (which is shown by a cross in Fig. 12) indicates that the gondola is just above the sea level. The gondola most likely sank afterward. Air pressure and temperature, as well as helium superpressure inside the balloon reported by flight 11 during its transit over the peninsula, are shown in Fig. 13. The crossing of the peninsula mountains is associated with huge fluctuations of temperature and pressure with, respectively, $17 \mathrm{~K}$ and 18 -hPa peak-topeak amplitudes. In the center of the disturbance, the balloon performed a vertical excursion of $1500 \mathrm{~m}$ in 15 min. Those pressure perturbations in return induced fluctuations of the helium superpressure inside the balloon, which reached $22.7 \mathrm{hPa}$ (i.e., a superpressure at the limit of what a $8.5-\mathrm{m}$ balloon can bear). This disturbance is most likely induced by a gravity wave generated by the tropospheric flow above the peninsula mountains that propagated upward in the stratosphere. Such large amplitude waves have already been reported at high latitudes above topography during winter (e.g., Dörnbrack et al. 1999; Hertzog et al. 2002a).

It is thus very likely that balloon 12 also crossed that disturbance $4 \mathrm{~h}$ after flight 11 . At that time, the sun began to rise and to heat the helium inside the balloon, so that the "background" superpressure inside balloon 12 already started to increase as it approached the mountains. Furthermore, balloon 12 is a larger balloon (10-m diameter) than balloon 11 and cannot bear superpressures larger than $20 \mathrm{hPa}$. Embedded in such a 

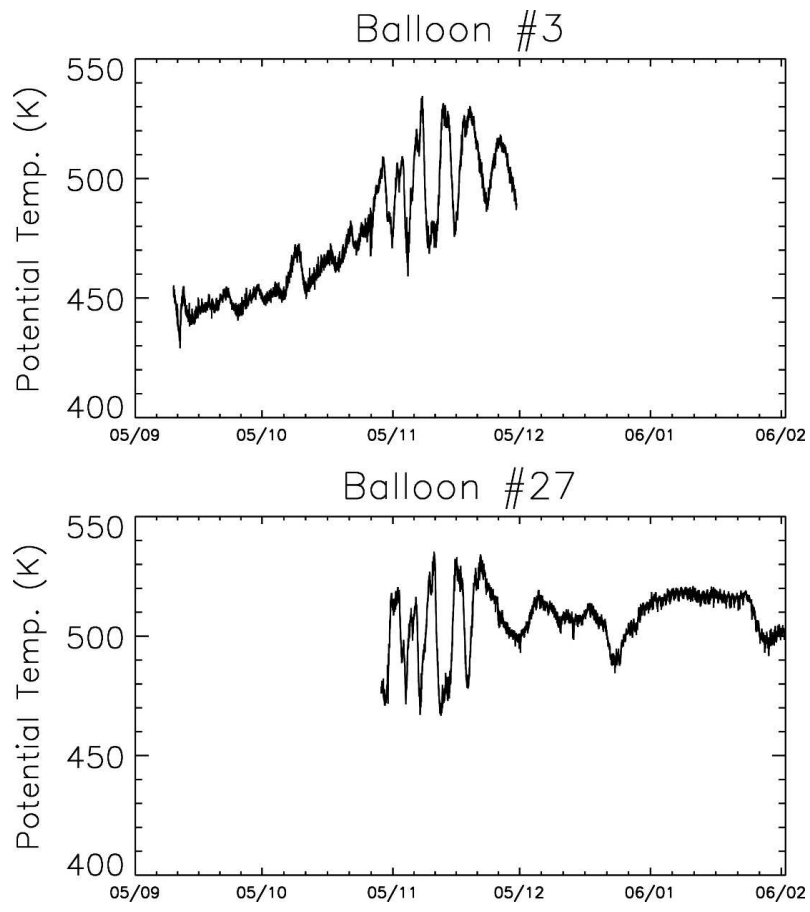

FIG. 11. (top) Air potential temperature measured along balloon 3 and (bottom) balloon 27 trajectories.

large amplitude mountain wave, balloon 12 certainly exploded over the peninsula and caused the fall of the gondola.

More generally, an increase of gravity wave activity

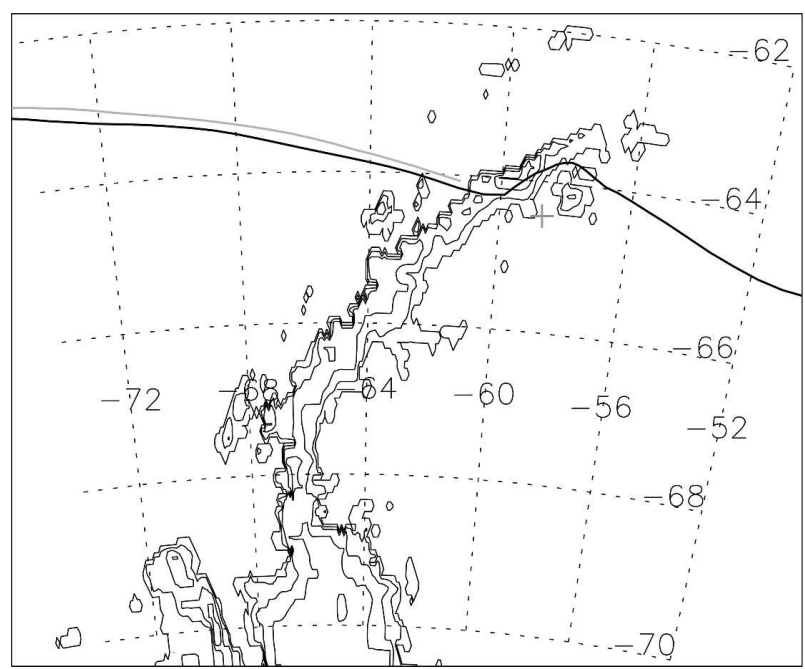

FIG. 12. Trajectories of flight 11 (black) and flight 12 (gray) on 6 and 7 Oct 2005. The last received position of flight 12, while the gondola was at $41 \mathrm{~m}$ ASL and descending over the parachute, is indicated by the gray cross on the eastern side of the peninsula. The elevation is represented by contours every $500 \mathrm{~m}$ from the sea level.
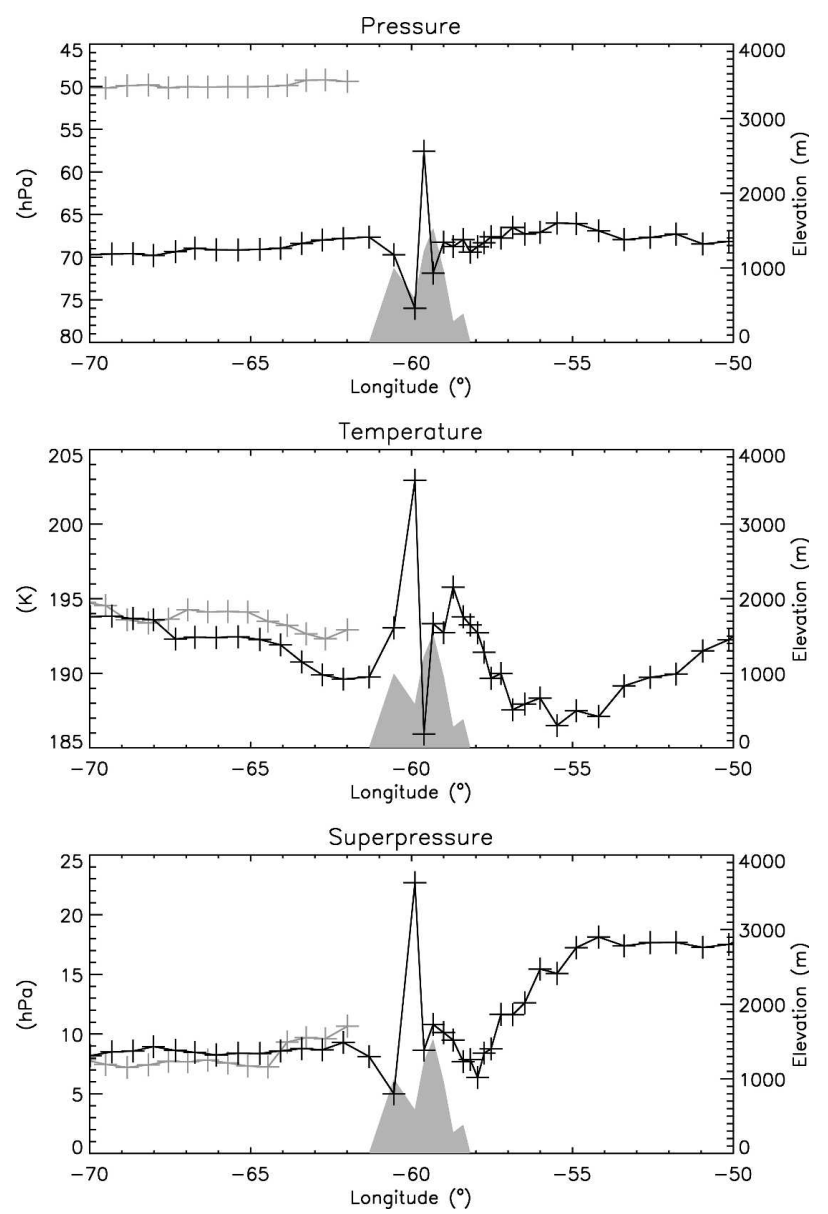

Fig. 13. (top) Air pressure, (middle) temperature, and (bottom) helium superpressure recorded with flight 11 (black) and flight 12 (gray) on 6-7 Oct 2005. The 15-min observations are shown with crosses. The gray shading at the bottom of each panel (right scale) represents the elevation overflown by flight 11 .

(with fortunately smaller amplitudes than those reported above) has been noticed in the Vorcore dataset when balloons were flying in the vicinity of steep topography (i.e., mainly the Antarctic Peninsula and the plateau-ocean transition). The dataset collected during Vorcore has a sufficient resolution to document those motions, and the geographical distribution of gravity waves over Antarctica that has been addressed by a few studies only (Allen and Vincent 1995; Pfenninger et al. 1999; Wu and Jiang 2002) will be the subject of forthcoming papers.

\section{Conclusions and future developments}

The Vorcore campaign thus proved that superpressure balloons can reliably perform long-duration flights in the very harsh environment typical of the polar lower 
stratosphere in winter. A mean flight duration of almost 2 months was achieved, and 16 flights out of 27 were actually longer that 2 months. The balloons were responsible for only $25 \%$ of the flight termination. Furthermore, thanks to modern satelliteborne communication and positioning systems, the dataset gathered during this campaign has typically the same size (and much better accuracy) as those of the early 1970s campaigns, although Vorcore only flew 27 balloons versus, for example, 480 during Éole.

Vorcore has provided a very valuable scientific dataset, which is original in many respects. For instance, its high resolution and global sampling of the SH polar stratosphere will be very helpful to document the dynamics of that region, from the short-period gravity waves up to the long Rossby planetary waves. Besides, the Vorcore observations were performed in a quasi-Lagrangian framework, which is a unique feature that is enabled by superpressure balloons. This characteristic is very useful to compute the momentum flux carried by gravity waves, and hence their potential forcing on the middle atmosphere (Hertzog and Vial 2001). With the help of ozone soundings performed along the balloon trajectories, the quasi-Lagrangian observations may also provide new insights on ozone depletion.

In the near future, several improvements of the observing system will be addressed in order to further increase its scientific interest. A higher sampling rate will be enabled by the use of satellite phone communications. New instruments aimed at measuring chemical species (e.g., ozone, water vapor) or particles and specially designed to bear the requirements of longduration flights in the lower stratosphere are under development. In parallel, larger balloons (12-m diameter) able to carry heavier payloads (up to $40 \mathrm{~kg}$ ) have already been successfully tested during tropical flights in 2006. Such new systems could be very useful to provide global-scale observations of still poorly sampled regions, such as, for instance, the equatorial lower stratosphere.

Acknowledgments. The Vorcore team is much indebted to R. Sadourny and H. Teitelbaum, who first proposed Stratéole, and more generally to all the scientists who supported the project through the ages. The authors are also grateful to the French Space Agency (CNES) and the Institut National des Sciences de l'Univers (INSU) for their longstanding support, and to the CNES, Zodiac, and CNRS staff involved in the project. The Vorcore campaign would not have been possible without the involvement of the NSF and of the French Polar Institute (IPEV). The whole NSF and RPSC teams in McMurdo are gratefully acknowledged for their outstanding help during the campaign. The Vorcore team would also like to thank T. Deshler and his team for their friendly support in McMurdo. Finally, the devotion of winter-over personnel who performed Vorcore-related observations in several Antarctic stations is gratefully acknowledged.

\section{REFERENCES}

Allen, S. J., and R. A. Vincent, 1995: Gravity wave activity in the lower atmosphere: Seasonal and latitudinal variations. $J$. Geophys. Res., 100, 1327-1350.

Angell, J. K., 1972: Air motions in the tropical stratosphere deduced from satellite tracking of horizontally floating balloons. J. Atmos. Sci., 29, 570-582.

Cocquerez, P., P. Guigue, R. Guilbon, T. Phulpin, M. Durand, J.-P. Lefèvre, M. Eymard, and M. Lafourcade, 2001: Test flights of CNES stratospheric superpressure balloons in experimental Arctic campaigns 2000-2001: Objectives and results. Proc. 15th ESA Symp. on European Rocket and Balloon Programmes and Related Research, SP-471, Noordwijk, Netherlands, European Space Agency, 597-601.

Deshler, T., M. E. Hervig, D. J. Hofmann, J. M. Rosen, and J. B. Liley, 2003: Thirty years of in situ stratospheric aerosol size distribution measurements from Laramie, Wyoming $\left(41^{\circ} \mathrm{N}\right)$, using balloon-borne instruments. J. Geophys. Res., 108, 4167, doi:10.1029/2002JD002514.

Dörnbrack, A., M. Leutbecher, R. Kivi, and E. Kyrö, 1999: Mountain-wave-induced record low stratospheric temperatures above northern Scandinavia. Tellus, 51A, 951-963.

Farman, J. C., B. G. Gardiner, and J. D. Shanklin, 1985: Large losses of ozone in Antarctica. Nature, 315, 207-210.

Fourrier, J., P. Morel, and P. Sitbon, 1970: Ambient temperature measurements from constant-level balloons. J. Appl. Meteor., 9, 154-157.

Hammam, A., 1991: Application des ballons à volume constant à l'étude de la basse atmosphère. Ph.D. thesis, Université Paul Sabatier, $210 \mathrm{pp}$.

Hertzog, A., and F. Vial, 2001: A study of the dynamics of the equatorial lower stratosphere by use of ultra-long-duration balloons, 2: Gravity waves. J. Geophys. Res., 106, 22745 22762 .

,-- _ A. Dörnbrack, S. D. Eckermann, B. M. Knudsen, and J.-P. Pommereau, 2002a: In situ observations of gravity waves and comparisons with numerical simulations during the SOLVE/THESEO 2000 campaign. J. Geophys. Res., 107, 8292, doi:10.1029/2001JD001025.

- - - C. R. Mechoso, C. Basdevant, and P. Cocquerez, 2002b: Quasi-Lagrangian measurements in the lower stratosphere reveal an energy peak associated with near-inertial waves. Geophys. Res. Lett., 29, 1229, doi:10.1029/ 2001GL014083.

— C. Basdevant, F. Vial, and C. R. Mechoso, 2004: The accuracy of stratospheric analyses in the Northern Hemisphere inferred from long-duration balloon flights. Quart. J. Roy. Meteor. Soc., 130, 607-626.

Koh, T.-Y., and A. Plumb, 2000: Lobe dynamics applied to barotropic Rossby-wave breaking. Phys. Fluids, 12, 1518-1528.

Lally, V. E., E. W. Lichfield, and S. B. Solot, 1966: The Southern Hemisphere GHOST experiment. WMO Bull., 15, 124-128.

Levanon, N., R. A. Oehlkers, S. D. Ellington, W. J. Massman, and 
V. E. Suomi, 1974: On the behavior of superpressure balloons at $150 \mathrm{mb}$. J. Appl. Meteor., 13, 494-504.

Massman, W. J., 1978: On the nature of vertical oscillations of constant volume balloons. J. Appl. Meteor., 17, 1351-1356.

McIntyre, M. E., 1989: On the Antarctic ozone hole. J. Atmos. Terr. Phys., 51, 29-43.

— , and T. N. Palmer, 1983: Breaking planetary waves in the stratosphere. Nature, 305, 593-600.

— mos. Terr. Phys., 46, 825-849.

Morel, P., and W. Bandeen, 1973: The EOLE experiment: Early results and current objectives. Bull. Amer. Meteor. Soc., 54, 298-306.

Moustaoui, M., H. Teitelbaum, and F. P. J. Valero, 2003: Ozone laminae inside the Antarctic vortex produced by poleward filaments. Quart. J. Roy. Meteor. Soc., 129, 3121-3136.

Nash, E. R., P. A. Newman, J. E. Rosenfield, and M. R. Schoeberl, 1996: An objective determination of the polar vortex using Ertel's potential vorticity. J. Geophys. Res., 101, 94719478.

Nastrom, G. D., 1980: The response of superpressure balloons to gravity waves. J. Appl. Meteor., 19, 1013-1019.

Olivero, J. J., A. W. Shaw, P. R. Williamson, and L. R. Megill, 1984: Mid-stratospheric circulations in the Southern Hemisphere from super pressure balloon trajectories. J. Geophys. Res., 89, 2595-2600.
Pfenninger, M., A. Z. Liu, G. C. Papen, and C. S. Gardner, 1999: Gravity wave characteristics in the lower atmosphere at South Pole. J. Geophys. Res., 104, 5963-5984.

Reynolds, R. D., 1973: Superpressure balloons as isentropic/ isopycnic tracers. J. Appl. Meteor., 12, 369-373.

Schumann, A. P., 1980: Carrier balloon trajectories in the stratosphere. J. Appl. Meteor., 19, 705-714.

The TWERLE Team, 1977: The TWERL Experiment. Bull. Amer. Meteor. Soc., 58, 936-948.

Vial, F., and Coauthors, 1995: STRATÉOLE: A project to study Antarctic polar vortex dynamics and its impact on ozone chemistry. Phys. Chem. Earth, 20, 83-96.

- A. Hertzog, C. R. Mechoso, C. Basdevant, P. Cocquerez, V. Dubourg, and F. Nouel, 2001: A study of the dynamics of the equatorial lower stratosphere by use of ultra-long-duration balloons, 1: Planetary scales. J. Geophys. Res., 106, 22 72522743.

Waugh, D. W., and Coauthors, 1994: Transport out of the lower stratospheric Arctic vortex by Rossby wave breaking. J. Geophys. Res., 99, 1071-1088.

WMO, 2003: Scientific assessment of ozone depletion: 2002. WMO/Global Ozone Research and Monitoring Project Rep. 47, 498 pp.

Wu, D. L., and J. H. Jiang, 2002: MLS observations of atmospheric gravity waves over Antarctica. J. Geophys. Res., 107, 4773, doi:10.1029/2002JD002390. 